\title{
The Association between Emergency Department Super-Utilizer Status and Willingness to Participate in Research
}

\author{
Henry W. Young II $\mathbb{D}^{1},{ }^{1}$ Emmett T. Martin, ${ }^{1}$ Evan Kwiatkowski, ${ }^{2}$ J. Adrian Tyndall ${ }^{1},{ }^{1}$ \\ and Linda B. Cottler ${ }^{3}$ \\ ${ }^{1}$ Department of Emergency Medicine, University of Florida, 1329 SW 16th Street, Suite 5270, Gainesville, FL 32610, USA \\ ${ }^{2}$ Department of Biostatics, University of North Carolina, 135 Dauer Drive, Chapel Hill, N.C. 27599, USA \\ ${ }^{3}$ Department of Epidemiology, College of Medicine and College of Public Health and Health Professions, University of Florida, \\ 2004 Mowry Road, Room 4218, P.O. Box 100231, Gainesville, FL 32610, USA
}

Correspondence should be addressed to Henry W. Young II; hyoungii@ufl.edu

Received 10 February 2020; Accepted 9 May 2020; Published 29 June 2020

Academic Editor: Jacek Smereka

Copyright (c) 2020 Henry W. Young II et al. This is an open access article distributed under the Creative Commons Attribution License, which permits unrestricted use, distribution, and reproduction in any medium, provided the original work is properly cited.

\begin{abstract}
Background. Research based on emergency departments (EDs) primarily focuses on medical conditions. There is limited research that investigates patients who willingly participate in research. This current study explored ED super-utilizers' (SUs') and nonsuper-utilizers' (NSUs') attitudes toward research. Objective. The study assesses the willingness of SUs to participate in research. We hypothesize that the SU population will be as interested as nonutilizers in participating in medical research. Methods. This prospective observational study stratified participants into SU and NSU cohorts based on their self-reported number of ED visits within 6 months. Surveys were captured in a secured database and analyzed using SAS 9.4. Results. 7,481 completed questionnaires. SUs were more interested in participating in all types of research compared to NSUs. Both groups were most willing to participate in surveys. Neither group was particularly interested in studies that required medications. SUs were not more willing to participate in studies without payment than NSUs. Both groups trusted researchers at the same rates. Conclusion. Although rarely included in medical research, SUs were more willing to participate in nearly all types of research and expressed a similar trust in medical research when compared to nonsuper-utilizers.
\end{abstract}

\section{Introduction}

Healthcare spending in the United States is at an all-time high. According to the Centers for Medicare \& Medicaid Services (CMS), in 2017, more than $\$ 3$ trillion dollars were spent on healthcare services in the United States. This was more than twice the amount spent in 2010 and represented $17.9 \%$ of the nation's gross domestic product [1]. The reason for rising healthcare costs is multifaceted and includes expansion of health insurance coverage under the Affordable Care Act and an increase in residential mental health and substance abuse facilities [2]. Studies have found that a large amount of healthcare resources are utilized by a small percentage of individuals [3]. The Agency for Healthcare Research and Quality found that 1\% of patients in the United
States were responsible for $21 \%$ of the entire healthcare spending in 2010 [4]. Due to rising healthcare costs, there has been a renewed national focus on reducing healthcare expenditures by developing and evaluating interventions and strategies targeted toward individuals who utilize healthcare services at higher rates than the general population [5-8].

Super-utilizers (SUs) are defined as individuals in healthcare who utilize a disproportionate amount of healthcare resources [4]. Among Medicaid patients, SUs were found to have more hospital stays, longer length of stays, and higher hospital costs per stay $[1,4]$. The 30 -day readmission rate was nearly 9 times higher for SUs than nonsuper-utilizers (NSUs). In the emergency department, SUs represent approximately $4.5 \%$ of all emergency 
department patients, but account for $28 \%$ of all emergency department visits [4]. This can be in part that SUs have more comorbidities and healthcare needs than NSUs $[4,9]$.

It has been stated in the past that this disproportionate utilization of resources may be due to unmet health needs and lack of access to appropriate settings. Due to the potential impact that SUs have on healthcare expenditures, continued research focused on this population is needed for more appropriate allocation of healthcare resources.

Very few studies evaluate the SU population outside the hospital from a community perspective, and no studies have assessed the interests of ED SUs to participate in research studies. The current analysis assesses the willingness of SUs in the community to participate in research, evaluate what type of research they would be interested in, and evaluate their trust in medical researchers. We hypothesize that the SU population will be as interested as nonutilizers in participating in medical research and trust in medical researchers would be no different than NSUs.

\section{Methods}

For this study, individuals were approached in community settings in North-Central and Northeast Florida through a community engagement program, HealthStreet. HealthStreet utilizes the community health worker (CHW) model to assess community members' health concerns and needs. CHWs approached individuals at public gathering places such as libraries, shopping centers, and community centers. Interested individuals were assessed with a University of Florida Institutional Review Board approved informed consent and a Health Intake Assessment. Within the Health Intake Assessment, individuals were asked about previous research experience, their interest in research, and their willingness to participate in research. Individuals were asked their willingness to participate in different types of studies including: surveys, studies requiring an overnight stay, studies that require providing blood samples including for genetic testing, and studies involving use of medical equipment. A commonly used definition of super-utilizers in the medical literature is an individual that averages $4 \mathrm{ED}$ visits per year [4]. Individuals that reported being evaluated in the emergency department 2 or more times over the last 6 months-which averages 4 visits per year-were identified as super-utilizers in this study. Data for this analysis were collected from October 2011 through December 2015. Group differences were analyzed using the chi-squared test for categorical variables and $t$-test for continuous variables. SAS 9.4 was used to analyze the data.

\section{Results}

Though CHWs contacted 9,134 individuals, informed consent was completed for 7,481. Among these individuals, CHWs interviewed 6,839 community members yielding a participation rate of $75 \%$. Approximately, $14 \%$ of the individuals included in the study met the criteria to be classified as SUs for a total of 962 individuals.

As seen in Table 1, SUs were more commonly female with an average age just over 40 years. SUs vs. NSUs did not differ by race or Latino/Hispanic ethnicity but did differ by marital status with SUs being more likely to be divorced, separated, or widowed ( $36 \%$ vs. $29 \%$; $p<0.0001)$.

SUs, compared to NSUs, were also more likely to be less educated, unemployed, have a higher average household size ( 3.3 vs. $3.1 ; p=0.03$ ), and report food insecurity (65\% vs. $43 \% ; p<0.0001)$. Importantly, SUs vs. NSUs were more likely to be uninsured ( $45.5 \%$ vs. $40.1 \% ; p<0.0001$ ) or have Medicaid or Medicare insurance (39.2\% vs. $36.1 \%$; $p<0.0001)$. The top health concerns were different between groups with the SUs being more concerned with conditions causing muscle and bone problems, weight problems, cancer, heart problems, and mental health conditions (see Table 2).

All participants were asked about their perceptions of medical research. Groups did not differ in prior participation in research; however, SUs were significantly more likely to be interested in participating in research (97\% vs. $92 \%$; $p<0.0001$ ) than NSUs.

Participants were assessed on their interests in specific study types including surveys, studies that only involved review of their medical records, studies that involved acquiring a blood sample, studies that involved genetic testing, studies that involved taking a medication, and those that involved staying overnight in the hospital or involved the use of medical equipment.

Overall, SUs were more interested in participating in all types of research compared to NSUs. Both groups were most willing to participate in surveys and least willing to volunteer in studies that required them to take medications. Although SUs were also more likely to say they would participate in every type of study, their willingness to participate in unpaid studies was the same as NSUs (79.6\% vs. $77.5 \% ; p=0.1453)$. Both groups trusted researchers and research at the same rates (see Table 3 ).

\section{Discussion}

To our knowledge, this is the first study of this size to assess ED SUs' interests in medical research. As we hypothesized, super-utilizers were not less trusting of medical researchers or medical research and more willing to participate in medical research than NSUs. This represents a large cohort of individuals that the literature has shown which is very dynamic and with more comorbidities than others who are interested and willing to participate in research despite few ever being involved in research $[10,11]$. This population also has unique health concerns that due to their low representation in medical research in the past may not be fully explored if they are not included in research.

Researching SUs presents a unique opportunity to learn more about individuals who utilize a disproportionate amount of healthcare resources. When compared to similar developed countries, the United States spends more of its gross domestic product on healthcare-related expenditures 
TABle 1: Demographics by emergency department utilization.

\begin{tabular}{|c|c|c|c|}
\hline & Super-utilizers $(N=962)$ & Nonsuper-utilizers $(N=5,877)$ & $p$ value \\
\hline \multicolumn{4}{|l|}{ Gender } \\
\hline Female & $63.7 \%$ & $56.2 \%$ & \multirow{2}{*}{$<0.0001$} \\
\hline Male & $36.3 \%$ & $43.8 \%$ & \\
\hline \multicolumn{4}{|l|}{ Average age } \\
\hline Female & 40.5 & 43.3 & 0.0001 \\
\hline Male & 45.9 & 42.8 & 0.0005 \\
\hline \multicolumn{4}{|l|}{ Race } \\
\hline Asian & $0.2 \%$ & $1.1 \%$ & \multirow{4}{*}{0.0603} \\
\hline African American & $62.3 \%$ & $62.6 \%$ & \\
\hline Caucasian & $30.7 \%$ & $29.7 \%$ & \\
\hline Other & $6.9 \%$ & $6.5 \%$ & \\
\hline Latino/hispanic* & $4.6 \%$ & $5.2 \%$ & 0.4311 \\
\hline \multicolumn{4}{|l|}{ Marital status } \\
\hline Never married & $49.1 \%$ & $49.7 \%$ & \multirow[t]{3}{*}{$<0.0001$} \\
\hline Married & $15.0 \%$ & $21.1 \%$ & \\
\hline Divorced/separated/widowed & $36.0 \%$ & $29.3 \%$ & \\
\hline $12+$ years of education & $71.4 \%$ & $79.8 \%$ & $<0.0001$ \\
\hline Currently employed & $21.6 \%$ & $36.8 \%$ & $<0.0001$ \\
\hline Veteran status & $10.0 \%$ & $10.0 \%$ & 0.9683 \\
\hline Household size (including participant) & 3.3 & 3.1 & 0.0384 \\
\hline Food insecure (not enough $\$$ to buy food) & $64.7 \%$ & $43.4 \%$ & $<0.0001$ \\
\hline \multicolumn{4}{|l|}{ Insurance status } \\
\hline Private insurance & $14.9 \%$ & $28.8 \%$ & \multirow{3}{*}{$<0.0001$} \\
\hline Medicaid/medicare & $39.6 \%$ & $31.2 \%$ & \\
\hline Uninsured & $45.5 \%$ & $40.1 \%$ & \\
\hline
\end{tabular}

TABle 2: Top 8 health concerns ${ }^{\dagger}$ by emergency department utilization.

\begin{tabular}{lccc}
\hline & Super-utilizers $(N=923)(\%)$ & Nonsuper-utilizers $(N=5,330)(\%)$ & $p$ value \\
\hline Hypertension & 30.6 & 31.1 & 0.7539 \\
Diabetes & 20.5 & 21.7 & 0.3870 \\
Muscle and bone problems & 25.2 & 18.2 & $<0.0001$ \\
Dental problems & 16.8 & 16.6 & 0.8756 \\
Weight problems & 12.4 & 16.4 & 0.0019 \\
Cancer & 11.6 & 15.7 & 0.0012 \\
Heart problems & 16.1 & 13.2 & 0.0180 \\
Mental health & 15.2 & 10.7 & $<0.0001$ \\
\hline
\end{tabular}

${ }^{\dagger}$ Among those with at least one health concern.

TABLE 3: Research perceptions by emergency department utilization.

\begin{tabular}{|c|c|c|c|}
\hline & $\begin{array}{c}\text { Super-utilizers } \\
(N=962) \\
\end{array}$ & $\begin{array}{c}\text { Nonsuper-utilizers } \\
(N=5,877)\end{array}$ & $p$ value \\
\hline Ever been in a health research study & $16.4 \%$ & $17.6 \%$ & 0.3387 \\
\hline Interested in participating in research & $97.2 \%$ & $92.1 \%$ & $<0.0001$ \\
\hline \multicolumn{4}{|l|}{ Would you volunteer for a health research study. . . } \\
\hline That only asked questions about your health? & $95.0 \%$ & $92.9 \%$ & 0.0168 \\
\hline If researchers wanted to see your medical records? & $91.0 \%$ & $85.3 \%$ & $<0.0001$ \\
\hline If you had to give a blood sample? & $90.1 \%$ & $83.8 \%$ & $<0.0001$ \\
\hline If you were asked to give a sample for genetic studies? & $87.0 \%$ & $83.2 \%$ & 0.0033 \\
\hline If you might have to take medicine? & $71.2 \%$ & $59.8 \%$ & $<0.0001$ \\
\hline If you were asked to stay overnight in a hospital or clinic? & $82.3 \%$ & $70.2 \%$ & $<0.0001$ \\
\hline If you might have to use medical equipment? & $87.9 \%$ & $81.1 \%$ & $<0.0001$ \\
\hline Would you participate in a study if you did not get paid? & $79.6 \%$ & $77.5 \%$ & 0.1453 \\
\hline Average trust in research ( $1-10$ scale, $1=$ "not at all," $10=$ completely) & 7.5 & 7.2 & 0.0624 \\
\hline $\begin{array}{l}\text { Average trust in researchers }(1-10 \text { scale, } 1=\text { "not at all," } \\
10=\text { completely })\end{array}$ & 7.4 & 7.2 & 0.1573 \\
\hline
\end{tabular}


than any other [4]. As health expenditures continue to increase at rates that are not sustainable, more research is needed to understand the needs of this population which will allow better care and decrease the need for unnecessary visits.

Health care disparities are defined by Healthy People 2020 as a particular type of health difference that is closely linked with social, economic, or environmental disadvantage [12]. In this study, the SUs had a greater rate of being food insecure, unemployed, less educated, and uninsured. Studies have demonstrated that individuals that are economically disadvantaged face barriers in accessing healthcare, present with more advanced disease, receive poorer quality of care, and are more likely to die of the disease and have worse health outcomes for acute and chronic conditions [13-16]. Multiple studies have demonstrated that insurance expansion associated with the Affordable Care Act has been associated with conflicting impacts on emergency department utilization among uninsured $[17,18]$. Although the degree to which these factors impact emergency department utilization is unclear, it does define this population as a vulnerable population. Due to these differences, SUs are at increased risk of suffering from health disparities which may lead to loss of opportunities to be in clinical studies. If SUs were more involved in medical research, then advancements could be made to reduce the need for these frequent emergency department visits while still meeting the needs of this vulnerable population.

The SU population includes individuals commonly underrepresented in medical research and thought to be difficult to recruit and retain in research $[19,20]$. Commonly cited barriers for both enrollment and retention in clinical trials include transportation, lack of access to primary care physicians, delayed diagnosis, multiple comorbidities which may exclude them from studies, and lack of appropriate childcare. Some studies suggest that due to difficulty in recruitment and retention of this population in biomedical research, studies may be disproportionately excluding this population [21, 22]. Similar to prior research, our study demonstrated that this cohort of SUs is as interested in participating in research as NSUs despite these barriers [23, 24].

Trust in research and medical researchers has been identified as a primary determinant in research participation [25-27]. A common cause of mistrust in research is perception of exploitation or negative past experiences with healthcare which is particularly high among racial and ethnic minorities [28]. Though mistrust is commonly identified as a barrier to research participation, trust in medical research and researchers is often cited as a significant facilitator for research participation [25-28]. Similarly, our sample of ED SUs was found to have high trust in medical research and willingness to participate in research. As this population represents an important component of the healthcare population, the addition of the super-utilizer population to medical research will assist with the generalizability of clinical studies and promote greater diversity in research participants.

The social factors among our ED SUs make this population economically disadvantaged and more susceptible to unethical practices within research and according to the "common rule" a vulnerable population. Compensation of individuals for their participation in research is a common practice that has been associated with increased recruitment rate and increased willingness to participate in research [29]. However, if payment for research is excessive relative to the risks of the study or what the individual is asked to do, then it can be coercive and unethical [30]. Despite these social factors, super-utilizers were not less willing to participate in research when payment was not provided, suggesting financial motivations are not the sole motivator for participation in research in this population.

Among our study population, SUs were found to have different health concerns than NSUs. In our cohort, SUs were more concerned about mental health, cardiovascular disease, and muscle or bone problems than NSUs. These conditions are all significant causes of morbidity and mortality in the US and are responsible for significant economic and societal burden. Among these diagnosis, significant health disparities exist including significant unmet need, poorer quality of care, and disparate healthcare utilization within these populations [31-33].

Limitations of the study include self-reported information which may suffer from recall bias regarding ED visits and conditions. However, the recall period was brief and the incident referred to a significant event (ED visits) that a person is likely to remember. Strengths of the study include a large diverse sample that was not restricted to one healthcare system.

\section{Conclusion}

Although rarely included in medical research, super-utilizers were more willing to participate in nearly all types of research and expressed a similar trust in medical research when compared to nonsuper-utilizers. Future research should consider the use of qualitative interviews to explore other factors that may contribute to SUs' attitude and willingness to participate in research and potential biases of researchers. Data from this research can assist in the development of targeted interventions to increase SUs' participation in research.

\section{Data Availability}

Data for this manuscript are available upon request.

\section{Conflicts of Interest}

The authors declare that they have no conflicts of interest.

\section{Acknowledgments}

The authors would like to acknowledge HealthStreet for their assistance with recruitment. This work was funded by the National Institute of Health (NIH)/National Institute on Drug Abuse (NIDA) (T32DA035167). 


\section{References}

[1] Center for Medicare \& Medicaid Services, NHE Fact Sheet, https://www.cms.gov/research-statistics-data-and-systems/ statistics-trends-and-reports/nationalhealthexpenddata/nhefact-sheet.html, Center for Medicare \& Medicaid Services, Baltimore, MD, USA, 2019, https://www.cms.gov/researchstatistics-data-and-systems/statistics-trends-and-reports/ nationalhealthexpenddata/nhe-fact-sheet.html.

[2] A. B. Martin, M. Hartman, B. Washington, and A. Catlin, "National health spending: faster growth in 2015 as coverage expands and utilization increases," Health Affairs, vol. 36, no. 1, pp. 166-176, 2017.

[3] J. J. G. Wammes, P. J. van der Wees, M. A. C. Tanke, G. P. Westert, and P. P. T. Jeurissen, "Systematic review of high-cost patients' characteristics and healthcare utilisation," BMJ Open, vol. 8, no. 9, p. e023113, 2018.

[4] H. J. Jiang, A. J. Weiss, M. L. Barrett, and M. Sheng, Characteristics of Hospital Stays for Super-Utilizers by Payer, 2012. HCUP Statistical Brief \#190, http://www.hcup-us.ahrq.gov/ reports/statbriefs/sb190-Hospital-Stays-Super-UtilizersPayer-2012.pdf, Agency for Healthcare Research and Quality, Rockville, MD, USA, 2015, http://www.hcup-us.ahrq.gov/ reports/statbriefs/sb190-Hospital-Stays-Super-UtilizersPayer-2012.pdf.

[5] S. Iovan, P. M. Lantz, K. Allan, and M. Abir, "Interventions to decrease use in prehospital and emergency care settings among super-utilizers in the United States: a systematic review," Medical Care Research and Review, vol. 77, no. 2, pp. 99-111, 2019.

[6] J. Bryk, G. S. Fischer, A. Lyons et al., "Improvement in quality metrics by the UPMC enhanced care program: a novel superutilizer program," Population Health Management, vol. 21, no. 3, pp. 217-221, 2018.

[7] A. B. Reedy-Cooper, C. Carmichael, J. L. Bowen et al., "Understanding perceived barriers to care among suburban super-utilizers who have an identified primary care provider," Population Health Management, vol. 23, no. 1, pp. 47-52, 2020.

[8] L. Hardin, S. Trumbo, and D. Wiest, "Cross-sector collaboration for vulnerable populations reduces utilization and strengthens community partnerships," Journal of Interprofessional Education \& Practice, vol. 18, Article ID 100291, 2020.

[9] L. J. Harris, I. Graetz, P. S. B. Podila, J. Wan, T. M. Waters, and J. E. Bailey, "Characteristics of hospital and emergency care super-utilizers with multiple chronic conditions," The Journal of Emergency Medicine, vol. 50, no. 4, pp. e203-e214, 2016.

[10] T. L. Johnson, D. J. Rinehart, J. Durfee et al., "For many patients who use large amounts of health care services, the need is intense yet temporary," Health Affairs, vol. 34, no. 8, pp. 1312-1319, 2015.

[11] R. G. Kronick, M. Bella, and T. P. Gilmer, The Faces of Medicaid III: Refining the Portrait of People with Multiple Chronic Conditions, Center for Health Care Strategies, Princeton, NJ, USA, 2009, http://www.chcs.org/media/Faces_ of_Medicaid_III.pdf.

[12] United States Department of Health and Human Services, Healthy People 2020, United States Department of Health and Human Services, Office of Disease Prevention and Health Promotion, Washington, DC, USA, 2020, https://www. healthypeople.gov/.
[13] J. M. McWilliams, "Health consequences of uninsurance among adults in the United States: recent evidence and implications," Milbank Quarterly, vol. 87, pp. 443-494, 2009.

[14] S. C. Markt, C. A. Lago-Hernandez, R. E. Miller et al., "Insurance status and disparities in disease presentation, treatment, and outcomes for men with germ cell tumors," Cancer, vol. 122, no. 20, pp. 3127-3135, 2016.

[15] J. Fang, G. Zhao, G. Wang, C. Ayala, and F. Loustalot, "Insurance status among adults with hypertension-the impact of underinsurance," Journal of the American Heart Association, vol. 5, no. 12, 2016.

[16] B. D. Sommers, A. A. Gawande, and K. Baicker, "Health insurance coverage and health-what the recent evidence tells us," New England Journal of Medicine, vol. 377, no. 6, pp. 586-593, 2017.

[17] R. Ladhania, A. M. Haviland, A. Venkat, R. Telang, and J. M. Pines, "The effect of Medicaid expansion on the nature of new enrollees' emergency department use," Medical Care Research and Review, 2019.

[18] H. Gotanda, G. Kominski, and Y. Tsugawa, "Association between the ACA medicaid expansions and primary care and emergency department use during the first 3 years," Journal of General Internal Medicine, vol. 35, no. 3, pp. 711-718, 2020.

[19] L. M. Perez and H. M. Treadwell, "Determining what we stand for will guide what we do: community priorities, ethical research paradigms, and research with vulnerable populations," American Journal of Public Health, vol. 99, no. 2, pp. 201-204, 2009.

[20] S. H.-X. Ng, N. Rahman, I. Y. H. Ang et al., "Characterization of high healthcare utilizer groups using administrative data from an electronic medical record database," BMC Health Services Research, vol. 19, no. 1, p. 452, 2019.

[21] K. Humphreys and C. Weisner, "Use of exclusion criteria in selecting research subjects and its effect on the generalizability of alcohol treatment outcome studies," American Journal of Psychiatry, vol. 157, no. 4, pp. 588-594, 2000.

[22] D. A. Webb, J. C. Coyne, R. L. Goldenberg et al., "Recruitment and retention of women in a large randomized control trial to reduce repeat preterm births: the Philadelphia collaborative preterm prevention project," BMC Medical Research Methodology, vol. 10, p. 88, 2010.

[23] J. Slomka, S. McCurdy, E. A. Ratliff, S. Timpson, and M. L. Williams, "Perceptions of financial payment for research participation among African-American drug users in HIV studies," Journal of General Internal Medicine, vol. 22, no. 10, pp. 1403-1409, 2007.

[24] D. F. Farmer, S. A. Jackson, F. Camacho, and M. A. Hall, "Attitudes of African American and low socioeconomic status white women toward medical research," Journal of Health Care for the Poor and Underserved, vol. 18, no. 1, pp. 85-99, 2007.

[25] G. L. Schmotzer, "Barriers and facilitators to participation of minorities in clinical trials," Ethnicity and Disease, vol. 22, no. 2, pp. 226-230, 2012.

[26] S. George, N. Duran, and K. Norris, "A systematic review of barriers and facilitators to minority research participation among African Americans, Latinos, Asian Americans, and Pacific Islanders," American Journal of Public Health, vol. 104, no. 2, pp. e16-e31, 2014.

[27] A. T. Limkakeng, L. L. H. de Oliveira, T. Moreira et al., "Systematic review and metasummary of attitudes toward research in emergency medical conditions," Journal of Medical Ethics, vol. 40, no. 6, pp. 401-408, 2014. 
[28] M. Smirnoff, I. Wilets, D. F. Ragin et al., "A paradigm for understanding trust and mistrust in medical research: the community VOICES study," AJOB Empirical Bioethics, vol. 9, no. 1, pp. 39-47, 2018.

[29] J. Brintnall-Karabelas, S. Sung, M. E. Cadman, C. Squires, K. Whorton, and M. Pao, "Improving recruitment in clinical trials: why eligible participants decline," Journal of Empirical Research on Human Research Ethics, vol. 6, no. 1, pp. 69-74, 2011.

[30] M. J. Czarny, N. E. Kass, C. Flexner, K. A. Carson, R. K. Myers, and E. J. Fuchs, "Payment to healthy volunteers in clinical research: the research subject's perspective," Clinical Pharmacology \& Therapeutics, vol. 87, no. 3, pp. 286-293, 2010.

[31] B. L. Cook, S. S.-Y. Hou, S. Y. Lee-Tauler, A. M. Progovac, F. Samson, and M. J. Sanchez, "A review of mental health and mental health care disparities research: 2011-2014," Medical Care Research and Review, vol. 76, no. 6, pp. 683-710, 2019.

[32] G. Graham, "Disparities in cardiovascular disease risk in the United States," Current Cardiology Reviews, vol. 11, no. 3, pp. 238-245, 2015.

[33] J. M. Jordan, R. Lawrence, R. Kington et al., "Ethnic health disparities in arthritis and musculoskeletal diseases: report of a scientific conference," Arthritis \& Rheumatism, vol. 46, no. 9, pp. 2280-2286, 2002. 